Voix et Images

voixetimages

\title{
Des provinciaux et des procrastinateurs
}

\section{Krzysztof Jarosz}

Volume 40, numéro 2 (119), hiver 2015

URI : https://id.erudit.org/iderudit/1030205ar

DOI : https://doi.org/10.7202/1030205ar

Aller au sommaire du numéro

Éditeur(s)

Université du Québec à Montréal

ISSN

0318-9201 (imprimé)

1705-933X (numérique)

Découvrir la revue

Citer ce compte rendu

Jarosz, K. (2015). Compte rendu de [Des provinciaux et des procrastinateurs].

Voix et Images, 40(2), 123-128. https://doi.org/10.7202/1030205ar

Ce document est protégé par la loi sur le droit d'auteur. L'utilisation des services d'Érudit (y compris la reproduction) est assujettie à sa politique d'utilisation que vous pouvez consulter en ligne.

https://apropos.erudit.org/fr/usagers/politique-dutilisation/
Cet article est diffusé et préservé par Érudit.

Érudit est un consortium interuniversitaire sans but lucratif composé de l’Université de Montréal, l'Université Laval et l'Université du Québec à Montréal. Il a pour mission la promotion et la valorisation de la recherche. https://www.erudit.org/fr/ 


\title{
E S S A I S / É T U D E S
}

Des provinciaux et des procrastinateurs

\author{
$+++$ \\ KRZYSZTOF JAROSZ \\ Université de Silésie
}

Ma lecture du livre de Jonathan Livernois Remettre à demain. Essai sur la permanence tranquille au Québec ${ }^{1}$ commence par une surprise. En évoquant le printemps érable de 2012, Livernois place, pour ainsi dire, en exergue du premier chapitre de son essai, un graffiti commémorant la réaction des manifestants face aux assauts de la police: «Duplessis reviens! T'as oublié tes chiens!» Et Livernois de poursuivre en disant que «les manifestations et la grogne populaire du printemps 2012 ont été marquées par une volonté, surprenante, de s'approprier le passé et ses symboles» (14): par exemple, entre autres, un portrait d'André Laurendeau, des fragments de poèmes de Gaston Miron, etc. Ce qui étonne, c'est que cette analogie ait été perçue par des jeunes, en dépit de ce qu'on diagnostique parfois comme une rupture de continuité intergénérationnelle, que certains, dans mon pays du moins, comparent à une coupure de continuité civilisationnelle, puisque la majorité des références culturelles de la génération des quinquagénaires ou sexagénaires polonais d'aujourd'hui diffèrent sensiblement de celles dans lesquelles se reconnaissent leurs enfants adultes. C'est donc finalement un bon signe que les jeunes Québécois manifestent (c'est le cas de le dire!) une telle connaissance de l'histoire de leur pays. Il est aussi somme toute réconfortant qu'à notre époque d'individualisme débridé - et parfois carrément paroxystique! - on soit encore capable de réagir en fonction d'un instinct grégaire, un peu trop injustement décrié. Cependant, l'évocation de la révolte estudiantine de 2012 n'est que l'introduction à la matière de l'essai dont l'argument n'est pas tout à fait inédit: Livernois aborde la question de ce qu'il appelle, d'après Pierre Vadeboncœur, «la permanence tranquille»: l'«impression d'être un peuple de tout repos et de toute éternité, [u]n peuple qui flotte sur l'Histoire et qui n'a pas besoin de se presser pour devenir ce qu'il est» (19). En d'autres termes, les Québécois sont (croient être) un peuple dont la pérennité est assurée quoi qu'il arrive, conviction qui - curieusement - coïncide avec des jérémiades qui prédisent la «louisianisation» du Québec. Ce sentiment de «fausse éternité» (21) fait que les Canadiens, les Canadiens français et les Québécois (donc les successifs avatars historiques d'un

1 Jonathan Livernois, Remettre à demain. Essai sur la permanence tranquille au Québec, Montréal, Boréal, $2014,146 \mathrm{p}$. 
même peuple) s'arrêtent juste avant de faire le pas décisif qui ferait d'eux une nation souveraine et libre. D'où vient cette tendance à la procrastination? Faut-il la chercher déjà dans le caractère provisoire des premières installations des colons français ayant échoué sur les berges du Saint-Laurent, et été constamment menacés d'anéantissement par les forces naturelles et hostiles, comme semble l'évoquer la phrase de Mère Catherine de Saint-Augustin, citée en exergue de l'essai: "Nous ne nous pressons pas pour achever le reste de nos bâtiments, à cause de l'incertitude où nous sommes si nous demeurerons longtemps ici...»? Toujours est-il que ce sont les événements ultérieurs qui ont formé ainsi les Québécois en gens qui se contentent du provisoire, à commencer par la Cession qui les a laissés pratiquement sans élites et sans le sentiment (vrai ou faux, n'importe) d'être protégés par leur lointain monarque, livrés à la bonne ou à la mauvaise volonté des occupants professant une autre religion et parlant une autre langue. Le repli autour du familier immédiat, c'est-à-dire l'esprit du clocher, est la réaction la plus naturelle. Chaque fois qu'ils sont obligés de sortir de l'intimité rassurante de leur paroisse, ils se voient économiquement et socialement subordonnés aux contremaîtres allophones, détenteurs du pouvoir et d'un savoir-faire exprimés dans une langue de prime abord incompréhensible qui finit par parasiter la langue maternelle de ces paysans dans tous les nouveaux secteurs qui dépassent le cadre de la vie quotidienne et des travaux agricoles. Le messianisme canadien qui va naître bientôt au confluent de ce repli sur soi d'un peuple ainsi conditionné et de l'idéologie ecclésiastique, sans doute elle-même écheveau inextricable d'un calcul politique et d'une foi authentique, va durablement marquer la mentalité canadienne-française. En abandonnant le pouvoir temporaire, tant politique qu'économique, aux «Anglais», qui, de toute façon, le détenaient de fait, et en se réservant une récompense eschatologique, on a créé un mécanisme infernal dont les principaux penseurs canadiens-français (et ensuite québécois) sont conscients. Parmi ceux que passe en revue Livernois, c'est peut-être Yvon Rivard qui résume le mieux ce trait de la mentalité québécoise, le dilemme qu'elle s'efforce en vain de trancher et, en même temps, l'objectif du livre de Livernois, qui consiste tant à décrire le phénomène dans la perspective historique qu'à tenter d'y chercher un remède, aussi difficile à trouver soit-il. Selon Rivard, donc: "Si nous avons un avenir, cet avenir ne peut être que le passé réécrit par ceux qui l'ont quitté et qui le réinventent, et ce passé, c'est l'héritage québécois de la pauvreté, l'héritage d'un peuple qui a appris, pour le meilleur et pour le pire, à se méfier des pouvoirs ${ }^{2}$.» Et Rivard (que je cite d'après Livernois) reprend dans un autre essai: «Ni français, ni américain, le Québécois francophone est le produit de cette double négation qui, en l'excluant en quelque sorte de l'histoire, ne lui a laissé aucune expérience du pouvoir et lui a légué une identité toute problématique ${ }^{3}$.» Séquelle de l'histoire de ce peuple nord-américain qui n'a pas oublié son ancienne patrie européenne, cette "procrastination tranquille» fonctionne même après la sortie de la Grande Noirceur, en freinant efficacement les tentatives successives des milieux indépendantistes. Livernois passe

2 Yvon Rivard, Une idée simple, Montréal, Boréal, coll. «Papiers collés», 2010, p. 69. Je cite d'après Jonathan Livernois, Remettre à demain, p. 116.

3 Yvon Rivard, Personne n'est une île, Montréal, Boréal, coll. «Papiers collés», 2006, p. 133. 
en revue les replis successifs (replis sur le plan des mentalités et des événements) qui suivent des élans d'énergie: la bataille gagnée de Saint-Denis suivie de la défaite à Saint-Charles et à Saint-Eustache, comme si les insurgés n'étaient pas prêts pour l'indépendance; la réclusion volontaire de Papineau dans des bibliothèques parisiennes (six mois à peine après cet effort armé) et bien d'autres faits plus au moins notoires. Livernois montre aussi des tentatives de sortir de ce moule fœtal dans lequel se pelotonnent les Québécois après chaque grand soulèvement collectif. Comme l'indique l'auteur, l'effort pour surmonter cette malédiction vient des milieux littéraires, or les exemples qu'il donne (Gérald Godin, Jacques Ferron, Gaston Miron, etc.) appartiennent à un passé héroïque et déjà révolu. Cependant, l'exemple du printemps érable permet de ne pas désespérer. Il est même, je l'ai dit, rassurant que les jeunes Québécois aient retrouvé, avec une telle facilité, intensité et célérité, des liens avec le passé, des analogies historiques qui témoignent de leur ancrage dans la mémoire nationale. Seulement, cela équivaut à reproduire le grand mécanisme cyclothymique à l'échelle nationale, la roue de l'éternel retour, la remise de l'entrée dans l'histoire au prochain épisode, à la prochaine fois... Cet enracinement dans le passé équivaut finalement à reproduire les réactions des générations précédentes. Dans Les noces, un drame du Polonais Stanisław Wyspiański, créé au début du xxe siècle, les convives qui représentent toutes les classes sociales, se mettent, comme dans une transe, à danser une ronde hypnotique, au lieu d'aller lutter pour l'indépendance du pays, comme ils avaient l'intention de le faire naguère encore pleins d'entrain. Je dédie cette image d'un passé heureusement révolu et surmonté aux amis québécois, tout en leur enviant une situation géopolitique qui leur permet de procrastiner à volonté à longueur des siècles, et je salue mon nouveau coéquipier, auteur de ce livre plein d'intêrêt qui constitue une excellente synthèse de la ronde québécoise de l'indécision.

Si l'essai de Jonathan Livernois a pour sujet les réactions collectives de tout un peuple, Mœurs de province ${ }^{4}$ de François Ricard est entièrement consacré aux réflexions d'un intellectuel individualiste qu'on n'imagine pas embrigadé à une cause, même s'il est loin d'être indifférent à ce qui se passe autour de lui, à l'image de $M$. Bergeret, professeur ami de la vérité, protagoniste inoubliable d'une tétralogie d'Anatole France. Ricard place son recueil d'essais et sa démarche d'observateur lucide et détaché sous le patronage de cette figure vénérable avec laquelle il s'identifie ouvertement. La «petite vieillesse» qu'avoue l'auteur de Mours de province, satisfait de son récent statut de professeur de littérature à la retraite, lui assure, dit-il, une «double liberté» (et une «double jouissance»): «celle de laisser braire et d'envoyer paître» (219). Si Ricard revendique un provincialisme de bon aloi, c'est que s'étant lui-même défini comme un provincial, il trouve cette situation assez confortable et même permettant un certain décentrement non seulement par rapport aux "centres» de plus en plus illusoires dans le monde d'aujourd'hui, mais aussi de l'idéologie mainstream de son milieu. Évidemment, même si son discours porte avant tout sur des problèmes

4 François Ricard, Mœurs de province, Montréal, Boréal, coll. «Papiers collés», 2014, 232 p. 
d'actualité, il n'oublie jamais son érudition, en incrustant ses chroniques de multiples références au professeur Bergeret, à Gustave Flaubert (à qui il emprunte le titre de son livre) et à Blaise Pascal, de qui Les Provinciales semble servir de vague modèle à Mœurs de province, ne serait-ce qu'à cause de la forme partiellement épistolaire du recueil de Ricard. Ce que Ricard ne dit pas, c'est que sa démarche est par moments proche de celle que Montesquieu fait pratiquer dans ses Lettres persanes à Uzbek et Rica. Pour en finir avec ces intertextes qui surgissent au détour de presque chaque phrase au grand plaisir du lecteur averti, évoquons la casuistique ${ }^{5}$ qui, comme méthode avouée ou non, accompagne la plupart des analyses auxquelles cet observateur perspicace soumet la réalité de sa province (et parfois du monde entier) en quête du loufoque, qui semble être sa catégorie fétiche, et qu'il détecte infailliblement là où ses congénères ne voient que du feu. Le farfelu (comme dirait André Malraux) n'apparaît qu'à ceux qui, comme Ricard, sont capables de voir au travers de cette carapace d'habitude qui rend invisible l'étrange, l'illogique, l'idéologique et finalement le risible du réel quotidien. Comme l'ironie est la figure la plus difficilement décelable quand l'intention du locuteur nous échappe, et puisque Ricard excelle à jongler entre un sérieux vrai et ce mode d'humour à froid qu'on appelle le pince-sans-rire, on a souvent du mal à interpréter son attitude envers les objets de ses chroniques. C'est le cas des considérations d'un sérieux à toute épreuve qu'il exhibe dans la chronique «Les règles de l'équité», où il soupèse et compare (sérieusement, demi-sérieusement, ou bien en se moquant in petto?) différentes catégories de minorités dont il faudrait égaliser les chances: "ne contribuerait-on pas davantage à l'éradication des inégalités et de l'uniformité qui affectent les campus en embauchant une lesbienne plutôt qu'une invalide, une invalide plutôt qu'une Algonkine, un Canado-Pakistanais plutôt qu'une femme? » (56) La conclusion qui invite les autorités à recourir à la casuistique est du même ordre: on ne sait pas si elle est ironique ou sérieuse. À l'époque actuelle où chacun tremble de peur qu'on ne lui intente un procès, les chroniques de Ricard sont parfaitement lisses; c'est en vain que les meilleurs des avocats y chercheraient une aspérité judiciairement utilisable, un crochet, une brèche. On a pourtant parfois l'impression qu'il choisit délibérément de mettre une plus forte touche d'ironie (ou peut-être d'accentuer sa distance) là où c'est moins dangereux, comme dans «Praline et les hassidim», texte qu'il consacre à la fameuse histoire d'un gymnase fréquenté par les bourgeoises francophones d'Outremont, situé face à une synagogue, différend qui a déclenché, je crois, la discussion sur les accommodements raisonnables. Or, si dans ce texte il y a un groupe presque ouvertement tourné en dérision, ce sont les Outremontaises d'un certain âge qui refusent de polir les vitres du gymnase au risque de polluer les rêves des garçons hassidim. Pour se convaincre qu'il y a dans ce recueil une intention parodique, il suffit de lire la chronique «Le grand humour».

5 En disséquant les catégories, les sous-catégories et les catégories mixtes de cas de minorités sexuelles, ethniques et autres afin d'établir les bases d'une politique d'équité, Ricard fait la remarque suivante avec une intention sans doute parodique: «Plus précisément, je dirais que, si nous disposons bel et bien, en ce domaine, d'une Morale générale très largement répandue et proclamée, ce qui nous fait de plus en plus cruellement défaut, c'est une bonne Casuistique, c'est-à-dire un code minutieux ou du moins une jurisprudence bien documentée qui nous permettrait de prendre les bonnes décisions au bon moment, sans léser personne ni risquer d'aggraver insidieusement les injustices que nous voulons à toute force corriger.» (57) 
Ricard s'y évertue à dresser une typologie de l'humour, en commençant par celui qui est évident, pratiqué par les humoristes professionnels, qui est une des marchandises québécoises d'exportation. Au deuxième degré se situe ce que Ricard appelle l'humour moyen,

qui demande déjà un certain effort de déchiffrement, et donc une sensibilité plus exercée. Car le comique, ici, ne se donne pas d'emblée; il est plutôt de l'ordre du double sens, de l'implicite, dissimulé entre les lignes ou à l'arrière-plan d'un texte qui, à première vue, se veut neutre, c'est-à-dire ni drôle ni sérieux, et soumis à une tout autre nécessité que la volontê humoristique. (62)

L'humour du troisième degré, que Ricard propose d'appeler «le grand humour», c'est la réaction qu'on a face à un texte écrit avec une intention sérieuse, mais tellement inintelligent que le prendre au sérieux relèverait, de la part du lecteur, d'un degré d'imbécillité aiguë, voire même incurable. "Y a-t-il quelque chose au-delà du grand humour? Un méta-humour encore plus épuré et plus drôle?", se demande Ricard et il se répond à lui-même: "Si oui, il ne peut être que le fait de l'Humoriste Suprême.» (69) Cet essai, en véritable mise en abyme explicative (si j'ose utiliser ce terme rébarbatif envers un si vénérable représentant des lettres classiques), situe la position de l'auteur entre (et contre) les tâcherons de l'humour farcesque et les agélastes, totalement dépourvus du sens de l'humour, qu'il fustige également. À la réflexion, je me demande si l'on ne pourrait appliquer à ce mode d'expression pince-sans-rire de Ricard l'hypothèse interprétative qu'Andrée Mercier a utilisée afin d'expliquer la manière d'écrire de Jacques Ferron. Or, si je me souviens bien de cette conclusion de Mercier, il faut lire les allégories de Ferron en même temps au degré littéral et au degré métaphorique. Par analogie, il se peut que Ricard ne soit pas forcément opposé au système d'égalisation des chances pour les minorités, mais, en même temps, il se rend compte des absurdités et des paradoxes de ce système lorsqu'on s'amuse à l'imaginer dans ce qu'il pourrait logiquement, mais invraisemblablement, être si on le poussait aux frontières de ses potentialités.

Ricard n'est pas tendre envers les poètes. En recourant à sa méthode favorite, que j'ai essayé de décrire plus haut, il fait une analyse sémantique d'un recueil de poèmes anonyme (mais, dit-il, existant bel et bien) foudroyante sur le plan du contenu. Je renvoie les lecteurs qui préfèrent la prose à la poésie à la chronique intitulée "Une soirée de lecture à la maison». C'est à se tordre de rire (si on s'est assuré au préalable qu'il n'y a aucun poète dans les parages!). D'ailleurs, en lisant le recueil, on s'aperçoit assez vite que la démarche de Ricard consiste dans une grande mesure à démythifier tout ce qu'il se met à observer. Je l'imagine se promenant avec une aiguille dont il use pour percer les ballons de la bêtise et de la grandiloquence de ses confrères intellectuels. Je lui voue surtout une admiration sans bornes pour son analyse de la «littérature-monde», une baliverne à laquelle ont peut-être même cru les signataires du célèbre manifeste (Pour une littérature-monde) et indubitablement certaines et certains de nos collègues «francophonistes» du monde entier qui se sont laissé duper par ce terme dont les (prétendus) effets n'ont pas duré plus longtemps qu'une saison éditoriale. 
J'estime aussi beaucoup Ricard pour sa vision lucide de la situation du français dans le monde et, plus particulièrement, au Québec. Versatile comme un caméléon, j'ai lu avec empathie et intérêt les pages que Livernois consacre au printemps érable, mais en passant au récit qu'en fait Ricard, l'intellectuel, l'individualiste, l'universitaire (et l'homme âgé) en moi n'a pas su réprimer un accès d'hilarité lorsque, en abordant le même événement, notre essayiste s'est mis à décrire

\begin{abstract}
un autre groupe [de manifestants], nullement opprimé [...] mais particulièrement influent (du moins en théorie): celui des professeurs, artistes, journalistes, intellectuels et autres manieurs de mots, tous plus ou moins protégés par l'État [...], et qui se sont lancés dans «la lutte» avec un enthousiasme et une rage auxquels nous n'étions plus habitués depuis très longtemps au Québec. (224)
\end{abstract}

Dans une autre de ses chroniques, Ricard parle avec réticence de la révolte estudiantine de mai 1968, en exprimant une sympathie pour les perdants, qui représentent pour lui un ancien monde, un «monde façonné par des siècles d'histoire, de culture, de pensée, d'humanité», "tout ce qui [...] faisait de l'individu autre chose qu'un être voué à la consommation et au bonheur» (205). Conservatisme? Certes! Un «âgisme» aigu (et j'entends par là le point de vue caractérisant les personnes matures aux opinions naturellement moins révolutionnaires que celles dont elles étaient peut-être elles-mêmes capables quand elles avaient quarante ans de moins qu'aujourd'hui)? Très probablement! Ce qui m'attire pourtant vers ce conservatisme lucide, c'est surtout une certaine honte que je ressens toujours lorsque je me retrouve en présence d'un esprit indépendant qui m'oblige à constater que l'opinion que j'avais jusqu'à ce moment-là sur un objet n'était en fait qu'un réflexe de suiveur qui se pelotonne confortablement et mollement dans le moule d'un avis général ou, en tout cas, admis dans le milieu où il évolue sans trop réfléchir, ni chercher à couper un cheveu en quatre.

Je dirais - sans aucunement improviser une «janusité» pour rationaliser ce que je viens de dire - que ces ouvrages me révèlent deux facettes de ma nature, conciliables dans un seul esprit d'une manière aussi incroyable que ces deux essais se côtoient dans cette chronique: l'un parle d'un réflexe spontané partagé par toute une communauté, et l'autre présente les réflexions d'un intellectuel animé du principe cartésien de tout examiner avant de formuler son jugement. Si j'imagine mal un Ricard (tel qu'il se dépeint dans son livre) s'abandonner au désordre des émotions grégaires, je crois ne pas être le seul à alterner des émois communautaires avec une réflexion critique, même si je suis incapable de m'élever à cet Olympe de la rationalité où siège François Ricard.

Ces deux essais sont parus au Boréal, comme certains autres dont j'avais parlé dans mes chroniques précédentes. Afin de ne pas être accusé de partialité, je promets de consacrer mon prochain texte aux livres publiés par d'autres éditeurs. 